\title{
Correction to: Continuous-Flow Microreactors for Polymer Synthesis: Engineering Principles and Applications
}

Yuanhai Su · Yang Song · Liang Xiang

\section{Correction to:}

Chapter 5 in: T. Noël, R. Luque (eds.), Accounts on Sustainable Flow Chemistry, Topics in Current Chemistry Collections, https://doi.org/10.1007/978-3-030-36572-1_5

This chapter was inadvertently published with incorrect heading levels, which has now been corrected. 